\title{
FLEXURAL VIBRATION AND BUCKLING ANALYSIS OF SINGLE-WALLED CARBON NANOTUBES USING DIFFERENT GRADIENT ELASTICITY THEORIES BASED ON REDDY AND HUU-TAI FORMULATIONS
}

\author{
DANILO KARLiČIĆ \\ Mathematical Institute of the Serbian Academy of Sciences and Arts, Belgrade, Serbia \\ Predrag Kozić, Ratko Pavlović \\ University of Nǐ̌, Department of Mechanical Engineering, Niš, Serbia \\ e-mail: kozicp@yahoo.com
}

\begin{abstract}
The aim of the present work is to analyze free flexural vibration and buckling of single-walled carbon nanotubes (SWCNT) under compressive axial loading based on different constitutive equations and beam theories. The models contain a material length scale parameter that can capture the size effect, unlike the classical Euler-Bernoulli or Reddy beam theory. The equations of motion of the Reddy and the Huu-Tai beam theories are reformulated using different gradient elasticity theories, including stress, strain and combined strain/inertia. The equations of motion are derived from Hamilton's principle in terms of the generalized displacements. Analytical solutions of free vibration and buckling are presented to bring out the effect of the nonlocal behavior on natural frequencies and buckling loads. The presented theoretical analysis is illustrated by a numerical example, and the results are qualitatively compared by another results.
\end{abstract}

Keywords: natural frequency, critical buckling load, gradient elasticity theories, nonlocal behavior

\section{Introduction}

Vibration and buckling problems of straight carbon nanotubes (CNT) (Spitalsky et al., 2010; Salvetat et al., 1999)occupy an important place in micro- and nano-scale devices and systems. Examples include nanosensors (Chopra et al., 2003, nanoactuators (Baughman et al., 1999, nanooscillators (Nishio et al., 2005), micro-resonators (Bak et al., 2008) and field emission devices (De Heer et al., 1995; Saito and Uemura, 2000), etc. In order to make full potential application of CNT, it is essential to understand their mechanical behavior well. In many papers, analytical analyses of the mechanical behavior of CNT have been proposed besides the experimental work by Ruoff et al. (2003). Carbon nanotubes can be modeled using atomistic (Zhang et al., 2005) or continuum mechanics methods ( $\mathrm{Li}$ and $\mathrm{Chou}, 2003)$. The atomic methods are limited to systems with a small number of molecules or atoms and therefore they are restricted to the study of small scale modeling. Unlike atomistic modeling, continuum models view CNT as a continuous beam. For realistic analysis of CNT, one must incorporate small-scale effects to achieve solutions with acceptable accuracy (Wang and Wang, 2007). Since the classical continuum models are scale free, for the modeling of CNT structures one can use modified elasticity theories like Eringen theory (Eringen, 1983; Eringen and Edelen, 1972) or strain gradient theories (Lam et al., 2003; Kong et al., 2009; Akgöz and Civalek, 2011). In this way, the internal size scale could be considered in the constitutive equation simply as a material parameter. In the theory of nonlocal elasticity, the stress at a reference point is considered to be a functional of the strain field at every point in the body. It can be concluded that continuum mechanics with size-effect could potentially 
play a useful role in the analysis related to nanostructures (Adali, 2012; Muc, 2011; Murmu and Adhikari, 2010a,b, 2011).

The first application of the Eringen nonlocal constitutive relation on the Euler-Bernoulli beam is the work of Peddinson et al. (2003). They investigated the deflection behavior of the nonlocal Euler-Bernoulli beam for different boundary conditions and possible application in microelectromechanical systems (MEMS). A new nonlocal shear deformation beam theory for bending, buckling and vibration of nanobeams was proposed by Huu-Tai (2012). The author derived the general equation of motion and took into account a quadratic variation of the shear strains across the thickness, based on nonlocal constitutive relation of Eringen. In the paper by Reddy and Pang (2008), the equations of motion of Euler-Bernoulli and Timoshenko beam theories were reformulated by the Eringen nonlocal theory, and then used to evaluate static bending, vibrations and buckling response of beams with various boundary conditions. Recently, nonlocal Euler-Bernoulli, Timoshenko, Reddy and Levinson beam theories were formulated by Reddy (2007) in a unified manner using Hamilton's principle and nonlocal elastic constitutive relation of Eringen. The analytical solution of natural frequency, critical buckling load and transversal deflection have been obtained for all presented beam theories. A comparison of stress gradient (Eringen's nonlocal theory) and two strain gradient theories applied to free vibration analysis of Euler-Bernoulli and Timoshenko beams was carried out by Ansari et al. (2012). Wang and Varadan (2006) studied the influence of scale-effect on natural frequencies and comparison with local natural frequencies of both single-walled CNT and double-walled CNT. They concluded that the classical continuum models are still valid and convenient for studying vibration responses of long and wide CNTs, especially for lower modes. The dynamic behavior of CNT embedded in an elastic medium (matrix) investigated by using nonlocal Timoshenko beam theory for both the stress gradient (Eringen nonlocal theory) and strain gradient approach were considered by Wang and Wang (2013). Their results show a significant dependence of frequencies on the surrounding medium and nonlocal parameter. The use of the nonlocal Timoshenko beam theory for analyzing free vibration and buckling behavior of nano-composite structures reinforced by single-walled carbon nanotubes (SWCNT) was proposed by Yas and Samadi (2012). They investigated the influence of geometrical and physical parameters such as nanotube volume fraction, foundation stiffness parameters, slenderness ratios and boundary conditions on the natural frequencies and critical buckling load. Liu and Reddy (2011) obtained a new model for static and free vibrations problems of a simply supported curved beam based on the nonlocal Timoshenko beam theory. Static and dynamic analyses of nanobeams based on the nonlocal Euler-Bernoulli, Timoshenko, Reddy, Levinson and Aydogdu beam theories were presented in Aydogdu (2009). The influence of the nonlocality and length of a nanobeam on natural frequencies, deflection and critical load were investigated in detail for each considered model. Based on the nonlocal elasticity and Euler-Bernoulli beam theory, the governing equation of transversal vibration of a nonuniform cantilever nanobeam was investigated by Murmu and Pradhan (2009). They obtained numerical results for the natural frequency from the governing equation by using the differential quadrature method and analyzing the influence of small-scale effects on the dynamic behavior of the nanocantilever.In the paper by Askes and Aifantis (2009), nonlocal and strain gradient elasticity theory were employed to obtain equations of motion for Euler-Bernoulli and Timoshenko beam theory. They investigated the influence of various material parameters of high order continuum theories on flexural wave dispersion in CNTs, and then compared the results with the results obtained by molecular dynamic (MD) simulations. Hosseini-Ara et al. (2012) proposed a new method to investigate the buckling behavior of short clamped CNTs, developed on the basis of the strain gradient theory and Timoshenko beam kinematics. They determined exact critical buckling loads using a linear polynomial and also investigated the influence of the scale coefficients, aspect ratio and transverse shear deformation on buckling of short clamped CNTs. Based on the strain gradient elasticity theory, the governing equation of motion for Euler-Bernoulli and 
higher shear deformation beam theories were derived by Akgöz and Civalek (2012, 2013). Also, they analyzed the influence of different material parameters on the dynamic and static behavior of a micro-size beam for different boundary conditions.

In the present paper, as an extension of the work by Ansari et al. (2012), we apply different gradient elasticity theories on the Reddy and Huu-Tai beam theories and obtain the governing equation for free flexural vibrations and buckling of SWCNT under axial loading. The discussions are limited only to the case of a simply supported straight nanotube. The natural frequencies and critical buckling load are obtained in the analytical form, based on Hamilton's principle by making use the stress gradient (nonlocal Eringen theory) and both strain gradient and combined strain/inertia gradient theories. The resulting equation for natural frequencies and critical buckling load contains a scale parameter and can capture the size effect. The differences between the natural frequencies and critical buckling load for stress gradient, strain gradient, combined strain/inertia theory and classical elasticity theory are shown and compared with the results by Ansari et al. (2012), and excellent agreement is shown.

\section{Structural model and theoretical formulation}

The Reddy and Huu-Tai beam theories are adopted in this study. These theories, which do not require shear correction factor, account for both small the scale effect and quadratic variation of shear strains and, consequently, shear stresses through thickness of the beam. In order to derive the equation of motions, we define the rectangular Cartesian coordinate system Oxyz. The $x$-coordinate is taken along the length of the beam, the $z$-coordinate along the thickness of the beam, and the $y$-coordinate along the width of the beam. We consider free vibration and buckling in the $x z$-plane.

\subsection{Constitutive relations}

According to the nonlocal theory, stress at a point depends not only on the strain at that point but also on strains at all other points of a body. The differential form of nonlocal constitutive relations for a one-dimensional structure was proposed by Eringen (1983) as

$$
\sigma_{x x}-\mu \frac{d^{2} \sigma_{x x}}{d x^{2}}=E \varepsilon_{x x} \quad \sigma_{x z}-\mu \frac{d^{2} \sigma_{x z}}{d x^{2}}=G \gamma_{x z}
$$

where $E$ and $G$ are the elastic modulus and shear modulus of the beam, respectively, $\mu=\left(e_{0} a\right)^{2}$ is the nonlocal parameter (length scales), $e_{0}$ is a constant to adjust the model to match the reliable results by experiments or microscopic models, $a$ is the internal characteristic length (e.g. lattice parameter, granular distance, wavelength) which can be identified from atomistic simulations or by using a dispersive curve of the Born-Karman model of lattice dynamics.

The combined strain/inertia constitutive relations for the one dimensional case, according to the papers by Ansari et al. (2012), Askes and Aifantis (2009) and Hosseini-Ara et al. (2012) are

$$
\sigma_{x x}=E\left(\varepsilon_{x x}+\mu \frac{d^{2} \varepsilon_{x x}}{d x^{2}}\right)+\rho \mu_{m} \ddot{\varepsilon}_{x x} \quad \sigma_{x z}=G\left(\gamma_{x z}+\mu \frac{d^{2} \gamma_{x z}}{d x^{2}}\right)+\rho \mu_{m} \ddot{\gamma}_{x z}
$$

where $\rho$ is the mass density and $\mu_{m}=l_{m}^{2}$ and $\mu=l^{2}$ are related to inertia gradients and strain gradient length scales, respectively. It should be noted that for $\mu_{m}=0$ the combined strain/inertia theories are reduced to strain gradient theories, and for $\mu=0$ the strain gradient theory is reduced to the classical elasticity theory. The inertia gradient length scale factors $\mu_{m}$, for the representative volume element (RVE) size, are related to the dynamic case, which tends to be larger than length scale factors $\mu$ for the static case. Accurate dynamical analysis of CNT 
is obtained using the inertia gradients length scales $\mu_{m}$. More details can be found in the paper by Askes and Aifantis (2009).

\subsection{The Reddy beam theory}

Based on the Reddy beam theory, the axial displacements $u(x, z, t)$ and transverse displacements $w(x, z, t)$ of any point of the beamare given by Reddy (2007) as

$$
\begin{aligned}
& u(x, z, t)=u_{0}(x, t)+z \phi_{x}(x, t)-c_{1} z^{3}\left(\phi_{x}(x, t)+\frac{\partial w_{0}(x, t)}{\partial x}\right) \\
& v(x, z, t)=0 \quad w(x, z, t)=w_{0}(x, t)
\end{aligned}
$$

where $c_{1}=4 /\left(3 h^{2}\right)$ and $h$ is the height of the beam, $w_{0}(x, t)$ and $\phi_{x}(x, t)$ are the transversal and rotation components of the displacement. The nonzero strains of the proposed beam theory are

$$
\begin{aligned}
\varepsilon_{x x} & =\frac{\partial u_{0}}{\partial x}+z \frac{\partial \phi_{x}}{\partial x}-c_{1} z^{3}\left(\frac{\partial \phi_{x}}{\partial x}+\frac{\partial^{2} w_{0}}{\partial x^{2}}\right) \\
\gamma_{x z} & =\left(1-c_{2} z^{2}\right)\left(\phi_{x}+\frac{\partial w_{0}}{\partial x}\right)
\end{aligned}
$$

where $c_{2}=3, c_{1}=4 / h^{2}$. In this case, the component $u_{0}$ of the axial displacement $u(x, z, t)$ is neglected.

Based on Hamilton's principle which states that the motion of an elastic structure during the time interval $0<t<T$ is such that the time integral of the total potential is extremum (Reddy, 2007) one writes

$$
\int_{0}^{T}(\delta U+\delta V-\delta K) d t=0
$$

where $\delta U$ is the variation of strain energy, $\delta V$ is the virtual work of external forces and $\delta K$ is the variation of kinetic energy of the nanobeam.

The variation of strain energy of the beam is

$$
\begin{aligned}
\delta U & =\int_{0}^{A} \int_{0}^{L}\left(\sigma_{x x} \delta \varepsilon_{x x}+\sigma_{x z} \delta \gamma_{x z}\right) d x d A \\
& =\int_{0}^{L}\left[\left(M_{x x}-c_{1} P_{x x}\right) \delta \frac{\partial \phi_{x}}{\partial x}-c_{1} P_{x x} \delta \frac{\partial^{2} w_{0}}{\partial x^{2}}+\left(Q_{x}-c_{2} R_{x}\right) \delta\left(\phi_{x}+\frac{\partial w_{0}}{\partial x}\right)\right] d x
\end{aligned}
$$

where $M_{x x}, P_{x x}, Q_{x}$ and $R_{x}$ are the stress resultants defined as

$$
\left(M_{x x}, P_{x x}\right)=\int_{0}^{A}\left(z, z^{3}\right) \sigma_{x x} d A \quad\left(Q_{x}, R_{x}\right)=\int_{0}^{A}\left(1, z^{2}\right) \sigma_{x z} d A
$$

The variation of potential energy of external forces can be expressed as

$$
\delta V=-\int_{0}^{L}\left(q(x) \delta w_{0}+\widehat{N}_{0} \frac{\partial w_{0}}{\partial x} \delta \frac{\partial w_{0}}{\partial x}\right) d x
$$

where $q(x)$ is the continual transversal load and $\widehat{N}_{0}$ is the axial load. 
The variation of kinetic energy is obtained as

$$
\begin{aligned}
\delta K & =\int_{0}^{A} \int_{0}^{L} \rho(\dot{u} \delta \dot{u}+\dot{w} \delta \dot{w}) d x d A=\int_{0}^{L}\left[\widehat{I}_{2} \dot{\phi}_{x} \delta \dot{\phi}_{x}-c_{1} \widehat{I}_{4}\left(\dot{\phi}_{x}+\frac{\partial \dot{w}_{0}}{\partial x}\right) \delta \dot{\phi}_{x}\right. \\
& \left.-c_{1} \widehat{I}_{4} \dot{\phi}_{x} \delta\left(\dot{\phi}_{x}+\frac{\partial \dot{w}_{0}}{\partial x}\right)+c_{1}^{2} \widehat{I}_{6}\left(\dot{\phi}_{x}+\frac{\partial \dot{w}_{0}}{\partial x}\right) \delta\left(\dot{\phi}_{x}+\frac{\partial \dot{w}_{0}}{\partial x}\right)+\widehat{I}_{0} \dot{w}_{0} \delta \dot{w}_{0}\right] d x
\end{aligned}
$$

where $\rho$ is the mass density and $\partial w_{0} / \partial t=\dot{w}_{0}$ is the time dderivative of the displacement $w_{0}$ and $\left(\widehat{I}_{0}, \widehat{I}_{2}, \widehat{I}_{4}, \widehat{I}_{6}\right)$ are the mass inertias defined as

$$
\left(\widehat{I}_{0}, \widehat{I}_{2}, \widehat{I}_{4}, \widehat{I}_{6}\right)=\int_{0}^{A}\left(1, z^{2}, z^{4}, z^{6}\right) \rho d A
$$

To derive the equations of motion associated with the present model, we substitute the expressions for $\delta U, \delta V$ and $\delta K$ from Eqs. (2.6), (2.8) and (2.9) into Eq. (2.5), and after integrating by parts and then collecting the coefficients of $\left.\delta w_{(} 0\right)$ and $\delta \phi_{x}$, the following equations of motion are obtained

$$
\begin{array}{ll}
\delta w_{0}: & \frac{\partial \bar{Q}_{x}}{\partial x}+c_{1} \frac{\partial^{2} P_{x x}}{\partial x^{2}}+\widehat{N}_{0} \frac{\partial^{2} w_{0}}{\partial x^{2}}=\widehat{I}_{0} \ddot{w}_{0}+\widehat{I}_{4} \frac{\partial \ddot{\phi}_{x}}{\partial x}-c_{1}^{2} \widehat{I}_{6}\left(\frac{\partial \ddot{\phi}_{x}}{\partial x}+\frac{\partial^{2} \ddot{w}_{0}}{\partial x^{2}}\right) \\
\delta \phi_{x}: & \frac{\partial \bar{M}_{x x}}{\partial x}-\bar{Q}_{x}=\ddot{\phi}_{x} \widehat{I}_{2}-c_{1} \widehat{I}_{4}\left(2 \ddot{\phi}_{x}+\frac{\partial \ddot{w}_{0}}{\partial x}\right)+c_{1}^{2} \widehat{I}_{6}\left(\ddot{\phi}_{x}+\frac{\partial \ddot{w}_{0}}{\partial x}\right)
\end{array}
$$

where

$$
\bar{Q}_{x}=Q_{x}-c_{2} R_{x} \quad \bar{M}_{x x}=M_{x x}-c_{1} P_{x x}
$$

The boundary conditions of the model are

$$
\begin{array}{lll}
w_{0} & \text { or } & V_{x}=c_{1} \frac{\partial P_{x x}}{\partial x}+\bar{Q}_{x}-\widehat{N}_{0} \frac{\partial w_{0}}{\partial x}-c_{1} \widehat{I}_{4} \ddot{\phi}_{x}+c_{1}^{2} \widehat{I}_{6}\left(\ddot{\phi}_{x}+\frac{\partial \ddot{w}_{0}}{\partial x}\right) \\
\frac{\partial w_{0}}{\partial x} & \text { or } & P_{x x} \\
\phi_{x} & \text { or } & \bar{M}_{x x}=M_{x x}-c_{1} P_{x x}
\end{array}
$$

where $V_{x}$ denotes the equivalent shear force.

By substituting Eq. (2.4) into Eq. (2.1) and the subsequent results into Eq. (2.7) and (2.12), the stress resultants for the nonlocal Eringen theory (stress gradient) are obtained as

$$
\begin{aligned}
& P_{x x}-\mu \frac{d^{2} P_{x x}}{d x^{2}}=E\left(I_{4}-c_{1} I_{6}\right) \frac{\partial \phi_{x}}{\partial x}-c_{1} I_{6} E \frac{\partial^{2} w_{0}}{\partial x^{2}} \\
& \bar{Q}_{x}-\mu \frac{d^{2} Q_{x}}{d x^{2}}=G \widehat{A}\left(\phi_{x}+\frac{\partial w_{0}}{\partial x}\right) \\
& \bar{M}_{x x}-\mu \frac{d^{2} \bar{M}_{x x}}{d x^{2}}=-E c_{1} I_{4}\left(2 \frac{\partial \phi_{x}}{\partial x}+\frac{\partial^{2} w_{0}}{\partial x^{2}}\right)+E I_{2} \frac{\partial \phi_{x}}{\partial x}+E c_{1}^{2} I_{6}\left(\frac{\partial \phi_{x}}{\partial x}+\frac{\partial^{2} w_{0}}{\partial x^{2}}\right)
\end{aligned}
$$

where

$$
\left(A, I_{2}, I_{4}, I_{6}\right)=\int_{0}^{A}\left(1, z^{2}, z^{4}, z^{6}\right) d A \quad \widehat{A}=\left(A-2 c_{2} I_{2}+c_{2}^{2} I_{4}\right)
$$


The equations of motion can be expressed in terms of the displacement $\left(w_{0}, \phi_{x}\right)$ for nonlocal constitutive relations (stress gradient). By substituting Eq. (2.14) into Eq. (2.11), we get the following equations of motion

$$
\begin{aligned}
& N_{0} \frac{\partial^{2} w_{0}}{\partial x^{2}}+\widehat{I}_{0} \ddot{w}_{0}-K_{1} \frac{\partial \ddot{\phi}_{x}}{\partial x}-c_{1}^{2} \widehat{I}_{6} \frac{\partial^{2} \ddot{w}_{0}}{\partial x^{2}}-\mu\left(N_{0} \frac{\partial^{4} w_{0}}{\partial x^{4}}+\widehat{I}_{0} \frac{\partial^{2} \ddot{w}_{0}}{\partial x^{2}}-K_{1} \frac{\partial^{3} \ddot{\phi}_{x}}{\partial x^{3}}-c_{1}^{2} \widehat{I_{6}} \frac{\partial^{4} \ddot{w}_{0}}{\partial x^{4}}\right) \\
& \quad=c_{1}\left[E\left(I_{4}-c_{1} I_{6}\right) \frac{\partial^{3} \phi_{x}}{\partial x^{3}}-c_{1} I_{6} E \frac{\partial^{4} w_{0}}{\partial x^{4}}\right]+G\left(\frac{\partial \phi_{x}}{\partial x}+\frac{\partial^{2} w_{0}}{\partial x^{2}}\right) \widehat{A} \\
& G\left(\phi_{x}+\frac{\partial w_{0}}{\partial x}\right) \widehat{A}+K_{2} \ddot{\phi}_{x}+K_{1} \frac{\partial \ddot{w}_{0}}{\partial x}-\mu\left(K_{2} \frac{\partial^{2} \ddot{\phi}_{x}}{\partial x^{2}}+K_{1} \frac{\partial^{3} \ddot{w}_{0}}{\partial x^{3}}\right) \\
& =E I_{2} \frac{\partial^{2} \phi_{x}}{\partial x^{2}}-E I_{4} c_{1}\left(2 \frac{\partial^{2} \phi_{x}}{\partial x^{2}}+\frac{\partial^{3} w_{0}}{\partial x^{3}}\right)+c_{1}^{2} E I_{6}\left(\frac{\partial^{2} \phi_{x}}{\partial x^{2}}+\frac{\partial^{3} w_{0}}{\partial x^{3}}\right)
\end{aligned}
$$

where $\widehat{N}_{0}=-N_{0}$ is the applied axial compressive force, and

$$
K_{1}=c_{1}^{2} \widehat{I}_{6}-c_{1} \widehat{I}_{4} \quad K_{2}=\widehat{I}_{2}-2 c_{1} \widehat{I}_{4}+c_{1}^{2} \widehat{I}_{6}
$$

In a very similar wayas in the previouscase, we get the stress resultants and equation of motion for the case of the combined strain/inertia gradient theory of elasticity. By substituting Eq. (2.4) into Eq. (2.2) and the subsequent results into Eqs. (2.7) and (2.12), the stress resultants for the combined strain/inertia gradient theory are obtained in the following form

$$
\begin{aligned}
P_{x x} & =E I_{4} \frac{\partial \phi_{x}}{\partial x}-c_{1} E I_{6}\left(\frac{\partial \phi_{x}}{\partial x}+\frac{\partial^{2} w_{0}}{\partial x^{2}}\right)+\mu E I_{4} \frac{\partial^{3} \phi_{x}}{\partial x^{3}}-\mu c_{1} E I_{6}\left(\frac{\partial^{3} \phi_{x}}{\partial x^{3}}+\frac{\partial^{4} w_{0}}{\partial x^{4}}\right) \\
& +\rho \mu_{m} I_{4} \frac{\partial \ddot{\phi}_{x}}{\partial x}-\rho \mu_{m} c_{1} I_{6}\left(\frac{\partial \ddot{\phi}_{x}}{\partial x}+\frac{\partial^{2} \ddot{w}_{0}}{\partial x^{2}}\right) \\
\bar{Q}_{x} & =G \widehat{A}\left(\phi_{x}+\frac{\partial w_{0}}{\partial x}\right)+\mu G \widehat{A}\left(\frac{\partial^{2} \phi_{x}}{\partial x^{2}}+\frac{\partial^{3} w_{0}}{\partial x^{3}}\right)+\rho \mu_{m} \widehat{A}\left(\ddot{\phi}_{x}+\frac{\partial \ddot{w}_{0}}{\partial x}\right) \\
\bar{M}_{x x} & =E I_{2} \frac{\partial \phi_{x}}{\partial x}-c_{1} E I_{4}\left(2 \frac{\partial \phi_{x}}{\partial x}+\frac{\partial^{2} w_{0}}{\partial x^{2}}\right)+\mu E I_{2} \frac{\partial^{3} \phi_{x}}{\partial x^{3}}-\mu c_{1} E I_{4}\left(\frac{\partial^{3} \phi_{x}}{\partial x^{3}}+\frac{\partial^{4} w_{0}}{\partial x^{4}}\right) \\
& +c_{1}^{2} E I_{6}\left(\frac{\partial \phi_{x}}{\partial x}+\frac{\partial^{2} w_{0}}{\partial x^{2}}\right)-\mu c_{1} E I_{4} \frac{\partial^{3} \phi_{x}}{\partial x^{3}}+\mu c_{1}^{2} E I_{6}\left(\frac{\partial^{3} \phi_{x}}{\partial x^{3}}+\frac{\partial^{4} w_{0}}{\partial x^{4}}\right)+\rho \mu_{m} I_{2} \frac{\partial \ddot{\phi}_{x}}{\partial x} \\
& -\rho \mu_{m} c_{1} I_{4}\left(2 \frac{\partial \ddot{\phi}_{x}}{\partial x}+\frac{\partial^{2} \ddot{w}_{0}}{\partial x^{2}}\right)+\rho \mu_{m} c_{1}^{2} I_{6}\left(\frac{\partial \ddot{\phi}_{x}}{\partial x}+\frac{\partial^{2} \ddot{w}_{0}}{\partial x^{2}}\right)
\end{aligned}
$$

By substituting Eq. (2.18) into Eq. (2.11), we obtain the following equation of motion for the combined strain/inertia gradients constitutive relation in terms of generalized displacements as

$$
\begin{aligned}
G & \widehat{A}\left(\frac{\partial \phi_{x}}{\partial x}+\frac{\partial^{2} w_{0}}{\partial x^{2}}\right)+\mu G \widehat{A}\left(\frac{\partial^{3} \phi_{x}}{\partial x^{3}}+\frac{\partial^{4} w_{0}}{\partial x^{4}}\right)+\rho \mu_{m} \widehat{A}\left(\frac{\partial \ddot{\phi}_{x}}{\partial x}+\frac{\partial^{2} \ddot{w}_{0}}{\partial x^{2}}\right)+c_{1} E I_{4} \frac{\partial^{3} \phi_{x}}{\partial x^{3}} \\
& -c_{1}^{2} E I_{6}\left(\frac{\partial^{3} \phi_{x}}{\partial x^{3}}+\frac{\partial^{4} w_{0}}{\partial x^{4}}\right)+\mu c_{1} E I_{4} \frac{\partial^{5} \phi_{x}}{\partial x^{5}}-\mu c_{1}^{2} E I_{6}\left(\frac{\partial^{5} \phi_{x}}{\partial x^{5}}+\frac{\partial^{6} w_{0}}{\partial x^{6}}\right)+\rho \mu_{m} c_{1} I_{4} \frac{\partial^{3} \ddot{\phi}_{x}}{\partial x^{3}} \\
& -\rho \mu_{m} c_{1}^{2} I_{6}\left(\frac{\partial^{3} \ddot{\phi}_{x}}{\partial x^{3}}+\frac{\partial^{4} \ddot{w}_{0}}{\partial x^{4}}\right)=N_{0} \frac{\partial^{2} w_{0}}{\partial x^{2}}+\widehat{I}_{0} \ddot{w}_{0}-K_{1} \frac{\partial \ddot{\phi}_{x}}{\partial x}-c_{1}^{2} \widehat{I}_{6} \frac{\partial^{2} \ddot{w}_{0}}{\partial x^{2}} \\
E I_{2} & \frac{\partial^{2} \phi_{x}}{\partial x^{2}}-c_{1} E I_{4}\left(2 \frac{\partial^{2} \phi_{x}}{\partial x^{2}}+\frac{\partial^{3} w_{0}}{\partial x^{3}}\right)+\mu E I_{2} \frac{\partial^{4} \phi_{x}}{\partial x^{4}}-\mu c_{1} E I_{4}\left(\frac{\partial^{4} \phi_{x}}{\partial x^{4}}+\frac{\partial^{5} w_{0}}{\partial x^{5}}\right) \\
& +c_{1}^{2} E I_{6}\left(\frac{\partial^{2} \phi_{x}}{\partial x^{2}}+\frac{\partial^{3} w_{0}}{\partial x^{3}}\right)-\mu c_{1} E I_{4} \frac{\partial^{4} \phi_{x}}{\partial x^{4}}+\mu c_{1}^{2} E I_{6}\left(\frac{\partial^{4} \phi_{x}}{\partial x^{4}}+\frac{\partial^{5} w_{0}}{\partial x^{5}}\right)+\rho \mu_{m} I_{2} \frac{\partial^{2} \ddot{\phi}_{x}}{\partial x^{2}} \\
& -\rho \mu_{m} c_{1} I_{4}\left(2 \frac{\partial^{2} \ddot{\phi}_{x}}{\partial x^{2}}+\frac{\partial^{3} \ddot{w}_{0}}{\partial x^{3}}\right)+\rho \mu_{m} c_{1}^{2} I_{6}\left(\frac{\partial^{2} \ddot{\phi}_{x}}{\partial x^{2}}+\frac{\partial^{3} \ddot{w}_{0}}{\partial x^{3}}\right)=G \widehat{A}\left(\phi_{x}+\frac{\partial w_{0}}{\partial x}\right) \\
& +\mu G \widehat{A}\left(\frac{\partial^{2} \phi_{x}}{\partial x^{2}}+\frac{\partial^{3} w_{0}}{\partial x^{3}}\right)+\rho \mu_{m} \widehat{A}\left(\ddot{\phi}_{x}+\frac{\partial \ddot{w}_{0}}{\partial x}\right)+K_{2} \ddot{\phi}_{x}+K_{1} \frac{\partial \ddot{w}_{0}}{\partial x}
\end{aligned}
$$


The equation of motion for the strain gradients constitutive relation, in terms of generalized displacements, is obtained from Eqs. (2.19) by setting the parameter $\mu_{m}$ equal to zero, as

$$
\begin{aligned}
G & \widehat{A} \\
& \left(\frac{\partial \phi_{x}}{\partial x}+\frac{\partial^{2} w_{0}}{\partial x^{2}}\right)+\mu G \widehat{A}\left(\frac{\partial^{3} \phi_{x}}{\partial x^{3}}+\frac{\partial^{4} w_{0}}{\partial x^{4}}\right)+c_{1} E I_{4} \frac{\partial^{3} \phi_{x}}{\partial x^{3}}-c_{1}^{2} E I_{6}\left(\frac{\partial^{3} \phi_{x}}{\partial x^{3}}+\frac{\partial^{4} w_{0}}{\partial x^{4}}\right) \\
& +\mu c_{1} E I_{4} \frac{\partial^{5} \phi_{x}}{\partial x^{5}}-\mu c_{1}^{2} E I_{6}\left(\frac{\partial^{5} \phi_{x}}{\partial x^{5}}+\frac{\partial^{6} w_{0}}{\partial x^{6}}\right)=N_{0} \frac{\partial^{2} w_{0}}{\partial x^{2}}+\widehat{I}_{0} \ddot{w}_{0}-K_{1} \frac{\partial \ddot{\phi}_{x}}{\partial x}-c_{1}^{2} \widehat{I}_{6} \frac{\partial^{2} \ddot{w}_{0}}{\partial x^{2}} \\
E I_{2} & \frac{\partial^{2} \phi_{x}}{\partial x^{2}}-c_{1} E I_{4}\left(2 \frac{\partial^{2} \phi_{x}}{\partial x^{2}}+\frac{\partial^{3} w_{0}}{\partial x^{3}}\right)+\mu E I_{2} \frac{\partial^{4} \phi_{x}}{\partial x^{4}}-\mu c_{1} E I_{4}\left(\frac{\partial^{4} \phi_{x}}{\partial x^{4}}+\frac{\partial^{5} w_{0}}{\partial x^{5}}\right) \\
& +c_{1}^{2} E I_{6}\left(\frac{\partial^{2} \phi_{x}}{\partial x^{2}}+\frac{\partial^{3} w_{0}}{\partial x^{3}}\right)-\mu c_{1} E I_{4} \frac{\partial^{4} \phi_{x}}{\partial x^{4}}+\mu c_{1}^{2} E I_{6}\left(\frac{\partial^{4} \phi_{x}}{\partial x^{4}}+\frac{\partial^{5} w_{0}}{\partial x^{5}}\right) \\
& =G \widehat{A}\left(\phi_{x}+\frac{\partial w_{0}}{\partial x}\right)+\mu G \widehat{A}\left(\frac{\partial^{2} \phi_{x}}{\partial x^{2}}+\frac{\partial^{3} w_{0}}{\partial x^{3}}\right)+K_{2} \ddot{\phi}_{x}+K_{1} \frac{\partial \ddot{w}_{0}}{\partial x}
\end{aligned}
$$

The equation of motion of the local beam theory can be obtained from Eq. (2.20) by setting the nonlocal parameter $\mu$ to be equal to zero.

\subsection{The Huu-Tai beam theory}

The displacement field of the Huu-Tai beam theory proposed by Huu-Tai (2012) is based on the following assumptions:

(1) the axial and transverse displacements consist of bending and shear components in which the bending components do not contribute toward shear forces and, likewise, the shear components do not contribute toward bending moments;

(2) the bending component of the axial displacement is similar to that given by the EulerBernoulli beam theory;

(3) the shear component of the axial displacement gives rise to the parabolic variation of the shear strain and hence to the shear stress through thickness of the beam in such a way that the shear stress vanishes on the top and the bottom surface.

According to these assumptions, we get the next displacement field

$$
\begin{aligned}
& u(x, z, t)=u_{0}(x, t)-z \frac{\partial w_{b}(x, t)}{\partial x}+\left[\frac{1}{4} z-\frac{5}{3} z\left(\frac{z}{h}\right)^{2}\right] \frac{\partial w_{s}(x, t)}{\partial x} \\
& v(x, z, t)=0 \quad w(x, z, t)=w_{b}(x, t)+w_{s}(x, t)
\end{aligned}
$$

whereh $h$ is the height of the beam, $w_{b}(x, t)$ and $w_{s}(x, t)$ are the bending and shear components of the transverse displacement and $u_{0}(x, t)$ is the axial displacement along the midplane of the beam. According to the proposed beam theory, the strain-displacement relations are given by

$$
\varepsilon_{x x}=\frac{\partial u_{0}}{\partial x}-z \frac{\partial^{2} w_{b}}{\partial x^{2}}-f \frac{\partial^{2} w_{s}}{\partial x^{2}} \quad \gamma_{x z}=g \frac{\partial w_{s}}{\partial x}
$$

where $f=-z / 4+5 z(z / h)^{2} / 3$ and $g=5 / 4-5(z / h)^{2}$. In this case, the component $u_{0}$ of the axial displacement $u(x, z, t)$ is neglected.

A nonlocal Huu-Tai beam model is developed using a different gradient elasticity theory and Hamilton's principle Eq. (2.5). The variation of strain energy of the Huu-Tai beam theory is

$$
\delta U=\int_{0}^{A} \int_{0}^{L}\left(\sigma_{x x} \delta \varepsilon_{x x}+\sigma_{x z} \delta \gamma_{x z}\right) d x d A=\int_{0}^{L}\left(-M_{b} \delta \frac{\partial^{2} w_{b}}{\partial x^{2}}-M_{s} \delta \frac{\partial^{2} w_{s}}{\partial x^{2}}+Q \delta \frac{\partial w_{s}}{\partial x}\right) d x
$$


where $M_{b}, M_{s}$ and $Q$ are the stress resultants defined as

$$
\left(M_{b}, M_{s}\right)=\int_{0}^{A}(z, f) \sigma_{x x} d A \quad Q=\int_{0}^{A} g \sigma_{x z} d A
$$

The variation of potential energy of external forces can be expressed as

$$
\delta V=-\int_{0}^{L}\left[q(x) \delta\left(w_{b}+w_{s}\right)+N_{0} \frac{\partial\left(w_{b}+w_{s}\right)}{\partial x} \delta \frac{\partial\left(w_{b}+w_{s}\right)}{\partial x}\right] d x
$$

where $q(x)$ is the continual transversal load and $N_{0}$ is the axial load.

The variation of kinetic energy is obtained as

$$
\begin{aligned}
\delta K & =\int_{0}^{A} \int_{0}^{L} \rho(\dot{u} \delta \dot{u}+\dot{w} \delta \dot{w}) d x d A \\
& =\int_{0}^{L}\left[\widehat{I}_{0}\left(\dot{w}_{b}+\dot{w}_{s}\right) \delta\left(\dot{w}_{b}+\dot{w}_{s}\right)+\widehat{I}_{2} \frac{\partial \dot{w}_{b}}{\partial x} \delta \frac{\partial \dot{w}_{b}}{\partial x}+\frac{\widehat{I}_{2}}{84} \frac{\partial \dot{w}_{s}}{\partial x} \delta \frac{\partial \dot{w}_{s}}{\partial x}\right] d x
\end{aligned}
$$

where $\widehat{I}_{0}$ and $\widehat{I}_{2}$ are defined in the preceding section, Eq. (2.10), $\dot{w}_{b}$ and $\dot{w}_{s}$ are time derivatives of the bending and shear components of the transverse displacement, respectively.

Substituting the expressions for $\delta U, \delta V$ and $\delta K$ from Eqs. (2.23), (2.25) and (2.26) into Hamilton's principle Eq. (2.5) and integrating by parts, and then collecting the coefficients of $\delta w_{b}$ and $\delta w_{s}$, we obtain

$$
\begin{array}{ll}
\delta w_{b}: & \frac{\partial^{2} M_{b}}{\partial x^{2}}-N_{0} \frac{\partial^{2}\left(w_{b}+w_{s}\right)}{\partial x^{2}}=\widehat{I}_{0}\left(\ddot{w}_{b}+\ddot{w}_{s}\right)-\widehat{I}_{2} \frac{\partial^{2} \ddot{w}_{b}}{\partial x^{2}} \\
\delta w_{s}: & \frac{\partial^{2} M_{s}}{\partial x^{2}}+\frac{\partial Q}{\partial x}-N_{0} \frac{\partial^{2}\left(w_{b}+w_{s}\right)}{\partial x^{2}}=\widehat{I}_{0}\left(\ddot{w}_{b}+\ddot{w}_{s}\right)-\frac{\widehat{I}_{2}}{84} \frac{\partial^{2} \ddot{w}_{s}}{\partial x^{2}}
\end{array}
$$

The boundary conditions involve specification of one element of each of the following four pairs at $x=0$ and $x=L$

$$
\begin{array}{lll}
w_{b} & \text { or } & V_{b}=\frac{\partial M_{b}}{\partial x}-N_{0} \frac{\partial\left(w_{b}+w_{s}\right)}{\partial x}+\widehat{I}_{2} \frac{\partial \ddot{w}_{b}}{\partial x} \\
w_{s} & \text { or } & V_{s}=\frac{\partial M_{s}}{\partial x}+Q-N_{0} \frac{\partial\left(w_{b}+w_{s}\right)}{\partial x}+\frac{\widehat{I}_{2}}{84} \frac{\partial \ddot{w}_{s}}{\partial x} \\
\frac{\partial w_{b}}{\partial x} & \text { or } & M_{b} \\
\frac{\partial w_{s}}{\partial x} & \text { or } & M_{s}
\end{array}
$$

where $V_{b}$ and $V_{s}$ are the equivalent transversal forces on the ends of the beam.

By substituting Eq. (2.22) into Eq. (2.1) and the subsequent results into Eq. (2.24), the stress resultants for the nonlocal Eringen theory (stress gradient) are obtained as

$$
\begin{aligned}
& M_{b}-\mu \frac{d^{2} M_{b}}{d x^{2}}=-E I_{2} \frac{d^{2} w_{b}}{d x^{2}} \quad M_{s}-\mu \frac{d^{2} M_{s}}{d x^{2}}=-\frac{E I_{2}}{84} \frac{d^{2} w_{s}}{d x^{2}} \\
& Q-\mu \frac{d^{2} Q}{d x^{2}}=\frac{5 G A}{6} \frac{\partial w_{s}}{\partial x}
\end{aligned}
$$

where $A$ and $I_{2}$ are defined in the previous section Eq. (2.15). 
By substituting Eq. (2.29) into Eq. (2.27), the nonlocal equations of motion can be expressed in terms of the displacements $w_{b}$ and $w_{s}$ as

$$
\begin{aligned}
- & E I_{2} \frac{\partial^{4} w_{b}}{\partial x^{4}}-N_{0}\left[\frac{\partial^{2}\left(w_{b}+w_{s}\right)}{\partial x^{2}}-\mu \frac{\partial^{4}\left(w_{b}+w_{s}\right)}{\partial x^{4}}\right] \\
= & \widehat{I}_{0}\left[\left(\ddot{w}_{b}+\ddot{w}_{s}\right)-\mu \frac{\partial^{2}\left(\ddot{w}_{b}+\ddot{w}_{s}\right)}{\partial x^{2}}\right]-\widehat{I}_{2}\left(\frac{\partial^{2} \ddot{w}_{b}}{\partial x^{2}}-\mu \frac{\partial^{4} \ddot{w}_{b}}{\partial x^{4}}\right) \\
- & \frac{E I_{2}}{84} \frac{\partial^{4} w_{s}}{\partial x^{4}}+\frac{5 G A}{6} \frac{\partial^{2} w_{s}}{\partial x^{2}}-N_{0}\left[\frac{\partial^{2}\left(w_{b}+w_{s}\right)}{\partial x^{2}}-\mu \frac{\partial^{4}\left(w_{b}+w_{s}\right)}{\partial x^{4}}\right] \\
= & \widehat{I}_{0}\left[\left(\ddot{w}_{b}+\ddot{w}_{s}\right)-\mu \frac{\partial^{2}\left(\ddot{w}_{b}+\ddot{w}_{s}\right)}{\partial x^{2}}\right]-\frac{\widehat{I}_{2}}{84}\left(\frac{\partial^{2} \ddot{w}_{s}}{\partial x^{2}}-\mu \frac{\partial^{4} \ddot{w}_{s}}{\partial x^{4}}\right)
\end{aligned}
$$

When the shear deformation effect of the nonlocal parameter is neglected $\left(w_{s}=0, \mu=0\right)$, the equations of motions Eq. (2.30) and boundary conditions in Eq. (2.28) are reduced to the Euler-Bernoulli beam theory.

The equation of motion for the case of the combined strain/inertia gradient theory of elasticity, according to the Huu-Tai beam theory, is obtained by substituting Eqs. (2.22) into Eqs. (2.2), then subsequent results into Eq. (2.24), the stress resultants are obtained as

$$
\begin{aligned}
& M_{b}=-E I_{2} \frac{\partial^{2} w_{b}}{\partial x^{2}}-\mu E I_{2} \frac{\partial^{4} w_{b}}{\partial x^{4}}-\rho \mu_{m} I_{2} \frac{\partial^{2} \ddot{w}_{b}}{\partial x^{2}} \\
& M_{s}=-\frac{E I_{2}}{84} \frac{\partial^{2} w_{s}}{\partial x^{2}}-\mu \frac{E I_{2}}{84} \frac{\partial^{4} w_{s}}{\partial x^{4}}-\rho \mu_{m} \frac{I_{2}}{84} \frac{\partial^{2} \ddot{w}_{s}}{\partial x^{2}} \\
& Q=\frac{5 G A}{6}\left(\frac{\partial w_{s}}{\partial x}+\mu \frac{\partial^{3} w_{s}}{\partial x^{3}}\right)+\rho \mu_{m} \frac{5 A}{6} \frac{\partial \ddot{w}_{s}}{\partial x}
\end{aligned}
$$

The equations of motion for the combined strain/inertia gradients constitutive relation, in terms of generalized displacements, are

$$
\begin{aligned}
& \widehat{I}_{0}\left(\ddot{w}_{b}+\ddot{w}_{s}\right)-\widehat{I}_{2} \frac{\partial^{2} \ddot{w}_{b}}{\partial x^{2}}+E I_{2} \frac{\partial^{4} w_{b}}{\partial x^{4}}+\mu E I_{2} \frac{\partial^{6} w_{b}}{\partial x^{6}}+\rho \mu_{m} I_{2} \frac{\partial^{4} \ddot{w}_{b}}{\partial x^{4}}+N_{0} \frac{\partial^{2}\left(w_{b}+w_{s}\right)}{\partial x^{2}}=0 \\
& \frac{5 G A}{6}\left(\frac{\partial^{2} w_{s}}{\partial x^{2}}+\mu \frac{\partial^{4} w_{s}}{\partial x^{4}}\right)+\rho \mu_{m} \frac{5 A}{6} \frac{\partial^{2} \ddot{w}_{s}}{\partial x^{2}}-N_{0} \frac{\partial^{2}\left(w_{b}+w_{s}\right)}{\partial x^{2}} \\
& \quad=\widehat{I}_{0}\left(\ddot{w}_{b}+\ddot{w}_{s}\right)-\frac{\widehat{I}_{2}}{84} \frac{\partial^{2} \ddot{w}_{s}}{\partial x^{2}}+\frac{E I_{2}}{84} \frac{\partial^{4} w_{s}}{\partial x^{4}}+\mu \frac{E I_{2}}{84} \frac{\partial^{6} w_{s}}{\partial x^{6}}+\rho \mu_{m} \frac{I_{2}}{84} \frac{\partial^{4} \ddot{w}_{s}}{\partial x^{4}}
\end{aligned}
$$

By setting the parameter $\mu_{m}$ equal to zero in Eqs. (2.32), we obtain the equation of motion for strain gradient constitutive equations as

$$
\begin{aligned}
& \widehat{I}_{0}\left(\ddot{w}_{b}+\ddot{w}_{s}\right)-\widehat{I}_{2} \frac{\partial^{2} \ddot{w}_{b}}{\partial x^{2}}+E I_{2} \frac{\partial^{4} w_{b}}{\partial x^{4}}+\mu E I_{2} \frac{\partial^{6} w_{b}}{\partial x^{6}}+N_{0} \frac{\partial^{2}\left(w_{b}+w_{s}\right)}{\partial x^{2}}=0 \\
& \frac{5 G A}{6}\left(\frac{\partial^{2} w_{s}}{\partial x^{2}}+\mu \frac{\partial^{4} w_{s}}{\partial x^{4}}\right)-N_{0} \frac{\partial^{2}\left(w_{b}+w_{s}\right)}{\partial x^{2}} \\
& \quad=\widehat{I}_{0}\left(\ddot{w}_{b}+\ddot{w}_{s}\right)-\frac{\widehat{I}_{2}}{84} \frac{\partial^{2} \ddot{w}_{s}}{\partial x^{2}}+\frac{E I_{2}}{84} \frac{\partial^{4} w_{s}}{\partial x^{4}}+\mu \frac{E I_{2}}{84} \frac{\partial^{6} w_{s}}{\partial x^{6}}
\end{aligned}
$$

The equation of motion of the local Huu-Tai beam theory can be obtained from Eq. (2.33) by setting the nonlocal parameter $\mu$ equal to zero.

\section{Analytical solutions of vibration and buckling of simply supported beams}

\subsection{The Reddy beam theory}

In the present work, a simply supported beam with length $L$, subjected to compressive axial loading $N_{0}$ is considered. The boundary conditions for the simply supported beam and the 
Reddy beam theory are

$$
w_{0}(0, t)=\bar{M}_{x x}(0, t)=P_{x x}(0, t)=w_{0}(L, t)=\bar{M}_{x x}(L, t)=P_{x x}(L, t)=0
$$

With the boundary conditions Eq. (3.1), Eqs. (2.16), (2.19) and (2.20) can be solved by assuming the solution in the following expansions of the generalized displacements $w_{0}$ and $\phi_{(}(x)$, as

$$
w_{0}(x, t)=\sum_{n=1}^{\infty} W_{n} \sin \alpha x \mathrm{e}^{\mathrm{i} \omega_{n} t} \quad \phi_{x}(x, t)=\sum_{n=1}^{\infty} X_{n} \cos \alpha x \mathrm{e}^{\mathrm{i} \omega_{n} t}
$$

where $\mathrm{i}=\sqrt{-1}, \alpha=n \pi / L,\left(W_{n}, X_{n}\right)$ are the amplitudes and $\omega_{n}$ is the natural frequency.

Substituting the expansions for $w_{0}$ and $\phi_{x}$ from Eqs. (3.2) into the equations of motion for different gradient Reddy beam models for SWCNT's, and solving the resulting eigenvalue problem, the natural frequency and critical buckling load of SWCNT's can be obtained

$$
\left(\left[\begin{array}{ll}
s_{11} & s_{12} \\
s_{21} & s_{22}
\end{array}\right]-\omega_{n}^{2}\left[\begin{array}{ll}
m_{11} & m_{12} \\
m_{21} & m_{22}
\end{array}\right]-N_{0}\left[\begin{array}{cc}
n_{11} & 0 \\
0 & 0
\end{array}\right]\right)\left\{\begin{array}{l}
W_{n} \\
X_{n}
\end{array}\right\}=\left\{\begin{array}{l}
0 \\
0
\end{array}\right\}
$$

where the coefficients for stress gradient theory are

$$
\begin{array}{ll}
s_{11}=E c_{1}^{2} I_{6} \alpha^{4}+G \alpha^{2} \widehat{A} & s_{12}=s_{21}=-E c_{1}\left(I_{4}-c_{1} I_{6}\right) \alpha^{3}+G \alpha \widehat{A} \\
s_{22}=G \widehat{A}+E\left(I_{2}-c_{1} I_{4}\right) \alpha^{2}-E c_{1}\left(I_{4}-c_{1} I_{6}\right) \alpha^{2} & \\
m_{11}=\widehat{I}_{0}+\alpha^{2} c_{1}^{2} \widehat{I}_{6}+\mu \alpha^{2} \widehat{I}_{0}+\mu \alpha^{4} c_{1}^{2} \widehat{I}_{6} & m_{12}=m_{21}=K_{1} \alpha+\mu K_{1} \alpha^{3} \\
m_{22}=K_{2}+\mu K_{2} \alpha^{2} & n_{11}=\alpha^{2}+\mu \alpha^{4}
\end{array}
$$

The coefficients in the strain/inertia gradient theory are

$$
\begin{aligned}
s_{11} & =G \alpha^{2} \widehat{A}+c_{1}^{2} E I_{6} \alpha^{4}-\mu G \alpha^{4} \widehat{A}-\mu c_{1}^{2} E I_{6} \alpha^{6} \\
s_{12} & =s_{21}=G \alpha \widehat{A}-\mu G \alpha^{3} \widehat{A}-E c_{1}\left(I_{4}-c_{1} I_{6}\right) \alpha^{3}+\mu E c_{1}\left(I_{4}-c_{1} I_{6}\right) \alpha^{5} \\
s_{22} & =G \widehat{A}-\mu G \widehat{A} \alpha^{2}+E\left(I_{2}-c_{1} I_{4}\right) \alpha^{2}-E c_{1}\left(I_{4}-c_{1} I_{6}\right) \alpha^{2} \\
& -E \mu\left(I_{2}-c_{1} I_{4}\right) \alpha^{4}+\mu E c_{1}\left(I_{4}-c_{1} I_{6}\right) \alpha^{4} \\
m_{11} & =\widehat{I}_{0}+\alpha^{2} c_{1}^{2} \widehat{I_{6}}+\rho \mu_{m} \widehat{A} \alpha^{2}+\rho \mu_{m} c_{1}^{2} I_{6} \alpha^{4} \\
m_{12} & =m_{21}=K_{1} \alpha+\rho \mu_{m} \widehat{A} \alpha-\rho \mu_{m} c_{1}\left(I_{4}-c_{1} I_{6}\right) \alpha^{3} \\
m_{22} & =K_{2}+\rho \mu_{m}\left(I_{2}-c_{1} I_{4}\right) \alpha^{2}-\rho \mu_{m} c_{1}\left(I_{4}-c_{1} I_{6}\right) \alpha^{2}+\rho \mu_{m} \widehat{A} \\
n_{11} & =\alpha^{2}
\end{aligned}
$$

The coefficients in the strain gradient theory $\left(\mu_{m}=0\right)$ are

$$
\begin{aligned}
s_{11} & =G \alpha^{2} \widehat{A}+c_{1}^{2} E I_{6} \alpha^{4}-\mu G \alpha^{4} \widehat{A}-\mu c_{1}^{2} E I_{6} \alpha^{6} \\
s_{12} & =s_{21}=G \alpha \widehat{A}-\mu G \alpha^{3} \widehat{A}-E c_{1}\left(I_{4}-c_{1} I_{6}\right) \alpha^{3}+\mu E c_{1}\left(I_{4}-c_{1} I_{6}\right) \alpha^{5} \\
s_{22} & =G \widehat{A}-\mu G \widehat{A} \alpha^{2}+E\left(I_{2}-c_{1} I_{4}\right) \alpha^{2}-E c_{1}\left(I_{4}-c_{1} I_{6}\right) \alpha^{2}-E \mu\left(I_{2}-c_{1} I_{4}\right) \alpha^{4} \\
& +\mu E c_{1}\left(I_{4}-c_{1} I_{6}\right) \alpha^{4} \\
m_{11} & =\widehat{I}_{0}+\alpha^{2} c_{1}^{2} \widehat{I}_{6} \quad m_{12}=m_{21}=K_{1} \alpha \quad m_{22}=K_{2} \quad n_{11}=\alpha^{2}
\end{aligned}
$$

The buckling load is obtained from Eq. (3.3) by setting $\omega_{n}$ to zero

$$
N_{0}=\frac{s_{11} s_{22}-s_{12}^{2}}{n_{11} s_{22}}
$$

The natural frequency is obtained from Eq. (3.3) by setting $N_{0}$ to zero

$$
\begin{aligned}
\omega_{n}^{2} & =\frac{1}{2\left(m_{11} m_{22}-m_{12}^{2}\right)}\left[m_{22} s_{11}-2 m_{12} s_{12}+m_{11} s_{22}\right. \\
& \left.-\sqrt{\left(-m_{22} s_{11}+2 m_{12} s_{12}-m_{11} s_{22}\right)^{2}-4\left(-m_{12}^{2}+m_{11} m_{22}\right)\left(-s_{12}^{2}+s_{11} s_{22}\right)}\right]
\end{aligned}
$$




\subsection{The Huu-Tai beam theory}

The simply supported beam boundary conditions for the Huu-Tai beam theory are

$$
\begin{aligned}
& w_{b}(0, t)=w_{s}(0, t)=M_{b}(0, t)=M_{s}(0, t)=w_{b}(L, t)=w_{s}(L, t)=M_{b}(L, t) \\
& \quad=M_{s}(L, t)=0
\end{aligned}
$$

With the governing boundary conditions Eq. (3.9), Eqs. (2.30), (2.32) and (2.33) can be solved by assuming the solution in the following expansions of the generalized displacements $w_{b}$ and $w_{s}$, as

$$
w_{b}(x, t)=\sum_{n=1}^{\infty} W_{b n} \sin \alpha x \mathrm{e}^{\mathrm{i} \omega_{n} t} \quad w_{s}(x, t)=\sum_{n=1}^{\infty} W_{s n} \sin \alpha x \mathrm{e}^{\mathrm{i} \omega_{n} t}
$$

where $\alpha=n \pi / L, W_{b n}$ and $W_{s n}$ are the amplitudes and $\omega_{n}$ is the natural frequency.

The closed-form solutions for the natural frequencies and buckling load of SWCNT using the Huu-Tai beam theory (Huu-Tai, 2012) for different gradients elasticity theories can be obtained by substituting the expansions of $w_{b}$ and $w_{s}$ into equations of motion Eqs. (2.30), (2.32) and (2.33), from the following equations

$$
\left(\left[\begin{array}{cc}
s_{11} & 0 \\
0 & s_{22}
\end{array}\right]-\omega_{n}^{2}\left[\begin{array}{ll}
m_{11} & m_{12} \\
m_{21} & m_{22}
\end{array}\right]-N_{0} n\left[\begin{array}{ll}
1 & 1 \\
1 & 1
\end{array}\right]\right)\left\{\begin{array}{l}
W_{b n} \\
W_{s n}
\end{array}\right\}=\left\{\begin{array}{l}
0 \\
0
\end{array}\right\}
$$

where the coefficients in the stress gradient theory are

$$
\begin{array}{lll}
s_{11}=E I_{2} \alpha^{4} & s_{22}=\frac{E I_{2}}{84} \alpha^{4}+\frac{5 G A}{6} \alpha^{2} & \lambda=1+\mu \alpha^{2} \\
m_{11}=\lambda\left(\widehat{I}_{0}+\alpha^{2} \widehat{I}_{2}\right) & m_{12}=m_{21}=\lambda \widehat{I}_{0} & m_{22}=\lambda\left(\widehat{I}_{0}+\alpha^{2} \frac{\widehat{I}_{2}}{84}\right) \\
n=\lambda \alpha^{2} & &
\end{array}
$$

Also, with the strain/inertia gradient theory one can obtain the coefficients

$$
\begin{array}{ll}
s_{11}=E I_{2} \alpha^{4} \lambda \quad s_{22}=\frac{E I_{2}}{84} \alpha^{4} \lambda+\frac{5 G A}{6} \alpha^{2} \lambda & \lambda=1-\mu \alpha^{2} \\
m_{11}=\widehat{I}_{0}+\alpha^{2} \widehat{I}_{2}+\rho \mu_{m} I_{2} \alpha^{4} & m_{12}=m_{21}=\widehat{I}_{0} \\
m_{22}=\widehat{I}_{0}+\alpha^{2} \frac{\widehat{I}_{2}}{84}+\rho \mu_{m} \frac{I_{2}}{84} \alpha^{4}+\rho \mu_{m} \frac{5 A}{6} \alpha^{2} & n=\alpha^{2}
\end{array}
$$

and in the strain gradient theory $\left(\mu_{m}=0\right)$

$$
\begin{array}{lll}
s_{11}=E I_{2} \alpha^{4} \lambda & s_{22}=\frac{E I_{2}}{84} \alpha^{4} \lambda+\frac{5 G A}{6} \alpha^{2} \lambda & \lambda=1-\mu \alpha^{2} \\
m_{11}=\widehat{I}_{0}+\alpha^{2} \widehat{I}_{2} & m_{12}=m_{21}=\widehat{I}_{0} & m_{22}=\widehat{I}_{0}+\alpha^{2} \frac{\widehat{I}_{2}}{84} \\
n=\alpha^{2} & &
\end{array}
$$

The buckling load is obtained from Eq. (3.11) by setting $\omega_{n}$ to zero

$$
N_{0}=\frac{s_{11} s_{22}}{n\left(s_{11}+s_{22}\right)}
$$

The natural frequency is obtained from Eq. (3.11) by setting $N_{0}$ to zero

$$
\omega_{n}^{2}=\frac{m_{22} s_{11}+m_{11} s_{22}-\sqrt{\left(m_{22} s_{11}+m_{11} s_{22}\right)^{2}-4\left(-m_{12}^{2}+m_{11} m_{22}\right) s_{11} s_{22}}}{2\left(-m_{12}^{2}+m_{11} m_{22}\right)}
$$




\section{Numerical results and discussion}

In the first part of this Section, we compare the obtained analytical results for both beam theories with the results previously published in literature. An excellent agreement is shown with the results obtained for the Timoshenko model of the nanobeam and also with the results obtained by MD simulation, proposed by Ansari et al. (2012). To investigate the effect of the nonlocal parameter and the aspect ratio on vibration and stability behavior of the Reddy and HuuTai beam for different constitutive relations, numerical analyses of the frequency and buckling load are performed and discussed. In what follows, we consider the armchair $(8,8)$ single-walled carbon nanotube $(\mathrm{SWCNT})$ with thickness $(h=0.34 \mathrm{~nm})$, Poisson's ratio $(\nu=0.3)$, mass density $\left(\rho=2300 \mathrm{~kg} / \mathrm{m}^{3}\right)$, Young's modulus $(E=1.1 \mathrm{TPa})$ and nonlocal parameters $(l=0.6 \mathrm{~nm}$, $\left.l_{m} / l=0.3\right)$. Numerical results for the natural frequency and buckling load of Reddy and Huu-Tai beam theories are presented in form of graphs and a table, using different constitutive relations, where influences of high order rotary inertias are also taken into account only to compute the natural frequencies.

\subsection{Validation study}

The results of fundamental natural frequencies of the simply supported SWCNT's for different constitutive relations are presented in Table 1 . In the presented analysis, the ratio of the dynamic and static length scale parameter $l_{m} / l$ is adopted from Ansari et al. (2012), which has obtained via MD simulations. The results obtained by presented analyses for different gradient theories for fundamental frequency are found to be in excellent agreementwith the one obtained from MD simulation and for the Timoshenko beam model shown by Ansari et al. (2012). From this table it can be seen that the fundamental frequencies decrease with the increasing beam aspect ratio for all cases of gradient elasticity and beam theories. Also, it is observed that the results obtained by applying the Huu-Tai and Reddy beam models lead to the identical results obtained for values of aspect ratios higher than 17.3. It is indicated that the influence of the nonlocal parameteris reflected in reduction of the fundamental frequency, but that it has no effect on the classical theory of elasticity. When the nonlocal parameter is sufficiently small, the discrepancy between various constitutive relations decreases so that the fundamental frequencies tend to converge at the fundamental frequencies in the classical elasticity theory. The comparison of the fundamental frequencies from the Reddy and Huu-Tai beam theory shows lower values in case of the combined strain/inertia gradient beam theory than for other gradient elasticity theories. Also, comparing the results for the combined strain/inertia gradient with the results obtained from MD simulation (Ansari et al., 2012), one can observe excellent agreement.

\subsection{Numerical examples}

Figure 1 shows the natural frequency from the Reddy and Huu-Tai beam theories as a function of the aspect ratio $(L / D)$ for various constitutive relations. It can be seen that the natural frequency decreases as the beam aspect ratio increases. When the beam aspect ratio is sufficiently larger, the discrepancy between various constitutive relations decreases so that the natural frequencies tend to converge to the aspect ratio. However, for small values of the aspect ratio, differences in the fundamental frequencies for different constitutive relations are much greater than for larger values. It should be noted that the natural frequency of the combined strain/inertia gradient beam theory is lower than those of other gradient elasticity theories and, therefore, this beam theory can accommodate the results of other ones for both cases. From the physical point of view, we can say that the influence of the aspect ratio $(L / D)$ on the dynamic behavior has damping effects. 
Table 1. Comparison of fundamental frequencies [THz] from different beam and gradient elasticity theories for $(8,8)$ armchair SWCNT's $\left(R / l=0.6 \mathrm{~nm}, l_{m} / l=8\right)$

\begin{tabular}{|c|c|c|c|c|c|c|c|c|c|c|c|c|c|}
\hline & \multicolumn{5}{|c|}{$\begin{array}{l}\text { Timoshenko beam theory } \\
\text { Ref. Ansari et al. (2012) }\end{array}$} & \multicolumn{4}{|c|}{ Reddy beam theory } & \multicolumn{4}{|c|}{ Huu-Tai beam theory } \\
\hline$L / D$ & $\begin{array}{c}\text { MD } \\
\text { simu- } \\
\text { lation }\end{array}$ & $\begin{array}{l}\text { Classical } \\
\text { theory }\end{array}$ & $\begin{array}{l}\text { Stress } \\
\text { gra- } \\
\text { dient } \\
\text { theory }\end{array}$ & $\begin{array}{l}\text { Strain } \\
\text { gra- } \\
\text { dient } \\
\text { theory }\end{array}$ & $\begin{array}{l}\text { Strain/ } \\
\text { inertia } \\
\text { gradient } \\
\text { theory }\end{array}$ & $\begin{array}{l}\text { Classical } \\
\text { theory }\end{array}$ & $\begin{array}{l}\text { Stress } \\
\text { gra- } \\
\text { dient } \\
\text { theory }\end{array}$ & $\begin{array}{l}\text { Strain } \\
\text { gra- } \\
\text { dient } \\
\text { theory }\end{array}$ & $\begin{array}{l}\text { Strain/ } \\
\text { inertia } \\
\text { gradient } \\
\text { theory }\end{array}$ & $\begin{array}{l}\text { Classical } \\
\text { theory }\end{array}$ & $\begin{array}{l}\text { Stress } \\
\text { gra- } \\
\text { dient } \\
\text { theory }\end{array}$ & $\begin{array}{l}\text { Strain } \\
\text { gra- } \\
\text { dient } \\
\text { theory }\end{array}$ & $\begin{array}{c}\text { Strain/ } \\
\text { inertia } \\
\text { gradient } \\
\text { theory }\end{array}$ \\
\hline 8.3 & 0.5299 & 0.5306 & 0.5302 & 0.5302 & 0.5299 & 0.5403 & 0.5393 & 0.5393 & 0.5387 & 0.5398 & 0.5387 & 0.5387 & 0.5381 \\
\hline 10.1 & 0.3618 & 0.3606 & 0.3604 & 0.3604 & 0.3603 & 0.3669 & 0.3664 & 0.3664 & 0.3662 & 0.3666 & 0.3661 & 0.3661 & 0.3659 \\
\hline 13.7 & 0.1931 & 0.1972 & 0.1971 & 0.1971 & 0.1971 & 0.2004 & 0.2003 & 0.2003 & 0.2002 & 0.2004 & 0.2002 & 0.2002 & 0.2002 \\
\hline 17.3 & 0.1103 & 0.1240 & 0.1240 & 0.1240 & 0.1240 & 0.1260 & 0.1259 & 0.1259 & 0.1259 & 0.1259 & 0.1259 & 0.1259 & 0.1259 \\
\hline 20.9 & 0.0724 & 0.0851 & 0.0851 & 0.0851 & 0.0851 & 0.0864 & 0.0864 & 0.0864 & 0.0864 & 0.0864 & 0.0864 & 0.0864 & 0.0864 \\
\hline 24.5 & 0.0519 & 0.0620 & 0.0620 & 0.0620 & 0.0620 & 0.0629 & 0.0629 & 0.0629 & 0.0629 & 0.0629 & 0.0629 & 0.0629 & 0.0629 \\
\hline 28.1 & 0.0425 & 0.0471 & 0.0471 & 0.0471 & 0.0471 & 0.0478 & 0.0478 & 0.0478 & 0.0478 & 0.0478 & 0.0478 & 0.0478 & 0.0478 \\
\hline 31.6 & 0.0358 & 0.0373 & 0.0373 & 0.0373 & 0.0373 & 0.0378 & 0.0378 & 0.0378 & 0.0378 & 0.0378 & 0.0378 & 0.0378 & 0.0378 \\
\hline 35.3 & 0.0287 & 0.0299 & 0.0299 & 0.0299 & 0.0299 & 0.0303 & 0.0303 & 0.0303 & 0.0303 & 0.0303 & 0.0303 & 0.0303 & 0.0303 \\
\hline 39.1 & 0.0259 & 0.0244 & 0.0244 & 0.0244 & 0.0244 & 0.0247 & 0.0247 & 0.0247 & 0.0247 & 0.0247 & 0.0247 & 0.0247 & 0.0247 \\
\hline
\end{tabular}



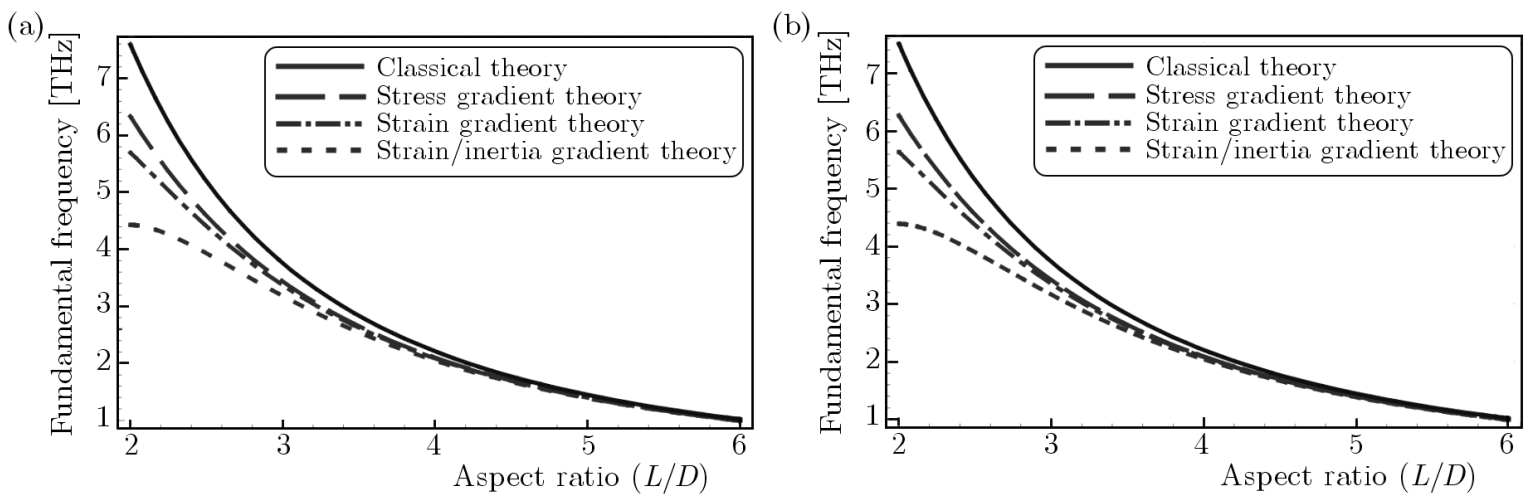

Fig. 1. Influence of the beam aspect ratio $(L / D)$ on various fundamental frequencies for $(8,8)$ armchair SWCNT's based on the (a) Reddy beam theory, (b) Huu-Tai beam theory; $l=0.6 \mathrm{~nm}, l_{m} / l=3$

The influence of the beam aspect ratio on the critical buckling load for various constitutive relations are shown in Fig. 2. It can be seen that the buckling load decreases with the increase in the beam aspect ratio for both beam theories. One can notice that small values of the beam aspect ratio $(L / D)$ cause significant differences in the buckling load for different constitutive relations. Also, the effect of the beam aspect ratio $(L / D)$ on the buckling load decreases with the increase in the aspect ratio. It can be concluded that the influences of $\mu$ on the critical buckling load are significant for small values of the aspect ratio and not for larger values. This conclusion also applies to the fundamental frequency. The buckling load for the combined strain/inertia gradients and strain gradients theory is the same for both beam theories, because $\mu_{m}$ does not affect the value of the buckling load.
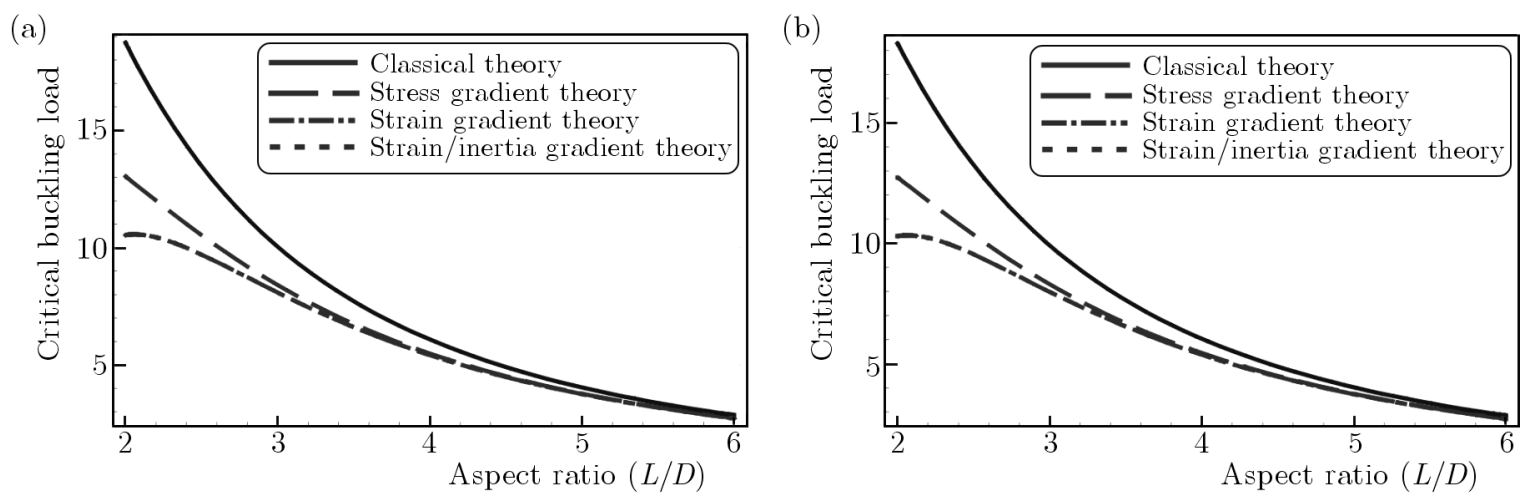

Fig. 2. Influence of the beam aspect ratio $(L / d)$ on various buckling loads for $(8,8)$ armchair SWCNT's based on the (a) Reddy beam theory, (b) Huu-Tai beam theory; $l=0.6 \mathrm{~nm}, l_{m} / l=3$

In order to investigate the effect of the nonlocal parameter on the natural frequency and buckling load of the SWCNT's, curves have been plotted as a function of the nonlocal parameter for four constitutive equations and both beam theories (Figs. 3 and 4). From Fig. 3 it can be seen that the increase in the nonlocal parameter causes a decrease in the natural frequency for all casesof constitutive relations. This implies that the overall stiffness of SWCNTs is significantly reduced due to the effects of the nonlocal parameter. From the physical point of view, we can say that the nonlocal parameter has a damping effect on the dynamic behavior of SWCNTs. Also, it is interesting to note that the values of the natural frequencyin the case of the strain/inertia gradient theory have the lowest value, which is in line with the previous results by Ansari et al. (2012). By comparing the values of the natural frequency from the Reddy (Fig. 3a) and Huu-Tai (Fig. 3a) beam theory, it can be concluded that the nonlocal parameter has a similar influence in both cases. Considering the influence of the nonlocal parameter on the buckling load (Fig. 4), 
a similar conclusion can be drawn as in the case of natural frequencies. Moreover, it can be seen that the strain/inertia gradient theory is reduced to the strain gradient theory, so it can be concluded that the inertia gradient length scale factors $\mu_{m}$ have no effect on the buckling load.

(a)

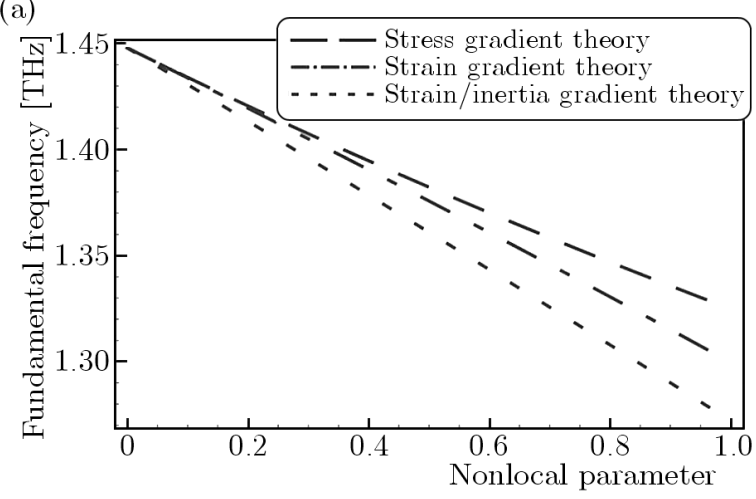

(b)

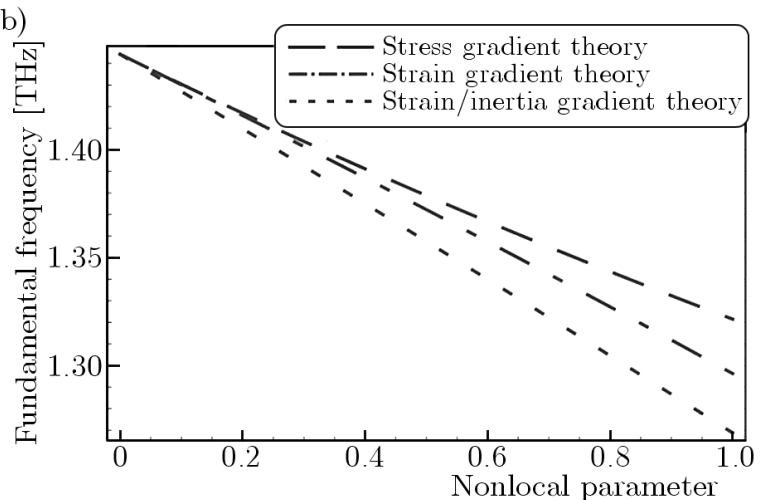

Fig. 3. Influence of the nonlocal parameter on various fundamental frequencies for $(8,8)$ armchair SWCNT's based on the (a) Reddy beam theory, (b) Huu-Tai beam theory; $L / d=5$
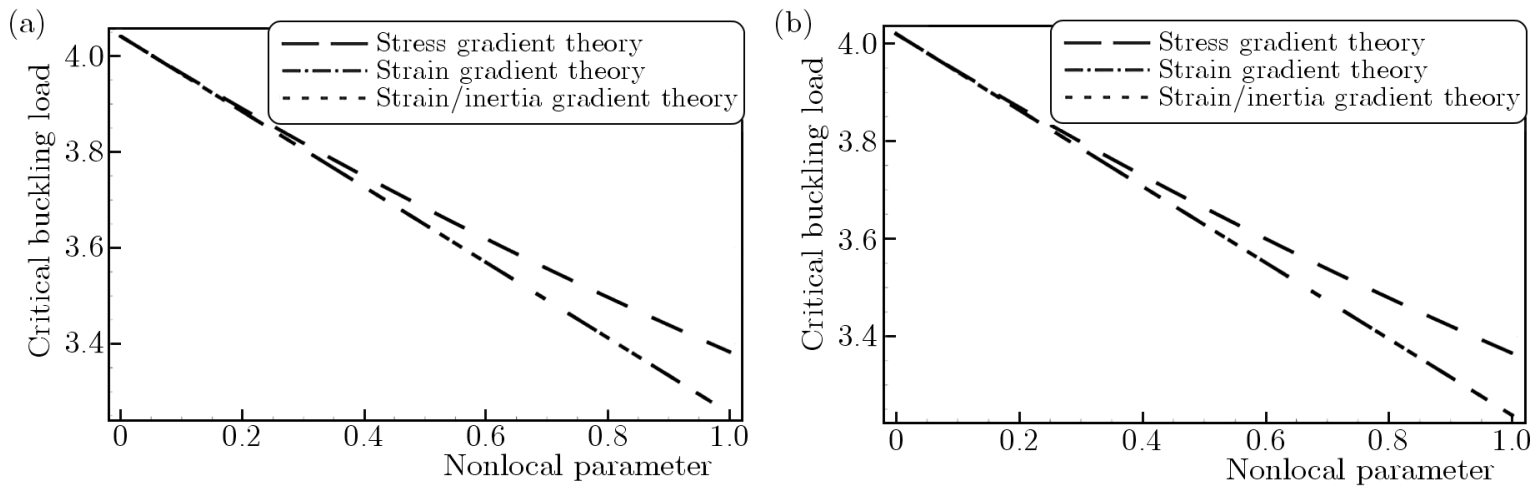

Fig. 4. Influence of the nonlocal parameter on various buckling loads for $(8,8)$ armchair SWCNT's based on the (a) Reddy beam theory and (b) Huu-Tai beam theory; $L / d=5$

\section{Conclusions}

In this paper, the influence of the aspect ratio and the nonlocal parameter on free vibration and buckling of SWCNT's based on different gradient elasticity theories is discussed. The Reddy and Huu-Tai beam theories have been utilized for modeling SWCNT's. The closed form solutions of natural frequencies and buckling load, which include the effect of the nonlocal parameter, shear deformation and rotary inertia, have been obtained for different constitutive relations. From the numerical results, it can be observed that the nonlocal parameter reduces the fundamental frequency and buckling load of simply supported SWCNT's. Also, it can be concluded that the aspect ratio increases the fundamental frequency but the buckling load decreases in both beam theories. Comparing these two beam theories, it is found that the fundamental frequency and buckling load predicted by the stress gradient, classical elasticity theories, combined strain/inertia and strain gradient elasticity are in good agreement. Also, the influence of nonlocal parameters $\mu$ is greater on the buckling load than on the fundamental frequencies. The dynamic nonlocal parameter $\mu_{m}$ does not affect the buckling load. The obtained analytical results have been compared with the results found in literature for the Timoshenko beam model and MD simulation, and excellent agreement is shown. 


\section{Acknowledgments}

This investigation has been supported by the research grants of the Serbian Ministry of Education, Science and Technological Development, number OI 174001 and OI 174011.

\section{References}

1. AdAli S., 2012, Variational formulation for buckling of multi-walled carbon nanotubes modelled as nonlocal Timoshenko beams, Journal of Theoretical and Applied Mechanics, 50, 1, 321-333

2. AkgÖz B., Civalek Ö., 2011, Strain gradient elasticity and modified couple stress models for buckling analysis of axially loaded micro-scaled beams, International Journal of Engineering Science, 49, $11,1268-1280$

3. Akgöz B., Civalek Ö., 2012, Analysis of micro-sized beams for various boundary conditions based on the strain gradient elasticity theory, Archive of Applied Mechanics, 82, 3, 423-443

4. Akgöz B., Civalek Ö., 2013, A size-dependent shear deformation beam model based on the strain gradient elasticity theory, International Journal of Engineering Science, 70, 1-14

5. Ansari R., Gholami R., Rouni H., 2012, Vibration analysis of single-walled carbon nanotubes using different gradient elasticity theories, Composites: Part B, 43, 2985-2989

6. Askes H., Aifantis E.C., 2009, Gradient elasticity and flexural wave dispersion in carbon nanotubes, Physical Review B: Condensed Matter and Materials Physics, 80, 195412

7. Aydogdu, M., 2009, A general nonlocal beam theory: its application to nanobeam bending, buckling and vibration, Physica E: Low-dimensional Systems and Nanostructures, 41, 9, 1651-1655

8. BaK J.H., Kim Y.D., Hong S.S., Lee B.Y., Lee S.R., Jang J.H., Park Y.D., 2008, Highfrequency micromechanical resonators from aluminium-carbon nanotube nanolaminates, Nature Materials, 7, 6, 459-463

9. Baughman R.H., Cui C., Zakhidov A.A., Iqbal Z., Barisci J.N., Spinks G.M., Kertesz M., 1999, Carbon nanotube actuators, Science, 284, 5418, 1340-1344

10. Chopra S., McGuire K., Gothard N., Rao A.M., Pham A., 2003, Selective gas detection using a carbon nanotube sensor, Applied Physics Letters, 83, 11, 2280-2282

11. De Heer W.A., Chatelain A., Ugarte D., 1995, A carbon nanotube field-emission electron source, Science, 270, 5239, 1179-1180

12. ERINGEN A.C., 1983, On differential equations of nonlocal elasticity and solutions of screw dislocation and surface waves, Journal of Applied Physics, 54, 9, 4703-4710

13. Eringen A.C., Edelen D.G.B., 1972, On nonlocal elasticity, International Journal of Engineering Science, 10, 3, 233-248

14. Hosseini-Ara R., Mirdamadi H.R., Khademyzadeh H., 2012, Buckling analysis of short carbon nanotubes based on a novel Timoshenko beam model, Journal of Theoretical and Applied Mechanics, 50, 4, 975-986

15. HuU-TAi T., 2012, A nonlocal beam theory for bending, buckling, and vibration of nanobeams, International Journal of Engineering Science, 52, 56-64

16. Kong S., Zhou S., Nie Z., WAng K., 2009, Static and dynamic analysis of micro beams based on strain gradient elasticity theory, International Journal of Engineering Science, 47, 4, 487-498

17. Lam D.C.C., Yang F., Chong A.C.M., Wang J., Tong P., 2003, Experiments and theory in strain gradient elasticity, Journal of the Mechanics and Physics of Solids, 51, 8, 1477-1508

18. Li C., Chou T.W., 2003, A structural mechanics approach for the analysis of carbon nanotubes, International Journal of Solids and Structures, 40, 10, 2487-2499

19. LiU Y.P., REDDy J.N., 2011, A nonlocal curved beam model based on a modified couple stress theory, International Journal of Structural Stability and Dynamics, 11, 3, 495-512 
20. Muc A., 2011, Modelling of carbon nanotubes behavior with the use of a thin shell theory, Journal of Theoretical and Applied Mechanics, 49, 2, 531-540

21. Murmu T., Adhikari S., 2011, Axial instability of double-nanobeam-systems, Physics Letters A, 375, 3, 601-608

22. Murmu T., Adhikari S., 2010a, Nonlocal transverse vibration of double-nanobeam-systems, Journal of Applied Physics, 108, 8, 083514

23. Murmu T., Adhikari S., 2010b, Nonlocal effects in the longitudinal vibration of double-nanorod systems, Physica E: Low-dimensional Systems and Nanostructures, 43, 1, 415-422

24. Murmu, T., Pradhan S.C., 2009, Small-scale effect on the vibration of nonuniformnanocantilever based on nonlocal elasticity theory, Physica E: Low-dimensional Systems and Nanostructures, 41, $8,1451-1456$

25. Nishio M., Sawaya S., Akita S., Nakayama Y., 2005, Carbon nanotube oscillators toward zeptogram detection, Applied Physics Letters, 86, 13, 133111

26. Peddieson J., Buchanan G.R., McNitt R.P., 2003, Application of nonlocal continuum models to nanotechnology, International Journal of Engineering Science, 41, 305-312

27. REDDy J.N., 2007, Nonlocal theories for buckling bending and vibration of beams, International Journal of Engineering Science, 45, 288-307

28. Reddy J.N., PAng S.D., 2008, Nonlocal continuum theories of beams for the analysis of carbon nanotubes, Journal of Applied Physics, 103, 023511

29. Ruoff R.S., QiAn D., Liu W.K., 2003, Mechanical properties of carbon nanotubes: theoretical predictions and experimental measurements, Comptes Rendus Physique, 4, 9, 993-1008

30. Saito Y., Uemura S., 2000, Field emission from carbon nanotubes and its application to electron sources, Carbon, 38, 2, 169-182

31. Salvetat J.P., Bonard J.M., Thomson N.H., Kulik A.J., Forro L., Benoit W., ZuppiROLI L., 1999, Mechanical properties of carbon nanotubes, Applied Physics A, 69, 3, 255-260

32. Spitalsky Z., Tasis D., Papagelis K., Galiotis C., 2010, Carbon nanotube-polymer composites: chemistry, processing, mechanical and electrical properties, Progress in Polymer Science, 35, $3,357-401$

33. WANG B.L., WANG K.F., 2013, Vibration analysis of embedded nanotubes using nonlocal continuum theory, Composites: Part B, 47, 96-101

34. WANG Q., VARADAN V.K., 2006, Vibration of carbon nanotubes studied using nonlocal continuum mechanics, Smart Materials and Structures, 15, 659-666

35. WANG Q., WANG C.M., 2007, The constitutive relation and small scale parameter of nonlocal continuum mechanics for modelling carbon nanotubes, Nanotechnology, 18, 7, 075702

36. YAs M.H., SAmAdi N., 2012, Free vibrations and buckling analysis of carbon nanotube-reinforced composite Timoshenko beams on elastic foundation, International Journal of Pressure Vessels and Piping, 98, 119-128

37. Zhang S., Mielke S.L., Khare R., Troya D., Ruoff R.S., Schatz G.C., Belytschko T., 2005, Mechanics of defects in carbon nanotubes: atomistic and multiscale simulations, Physical Review B, 71, 11, 115403 